BULL. AUSTRAL. MATH. SOC.

VOL. $24(1981), 93-122$.

\title{
DIFFERENTIAL CALCULUS \\ IN FRÉCHET SPACES
}

\author{
Duong Minh Duc
}

We apply Keller's method to the study of differential calculus in Frechet spaces and establish an inverse mapping theorem. A special case of this theorem is similar to a theorem of Yamamuro.

\section{Introduction}

Let $E$ and $F$ be two Fréchet spaces over the field $\mathbb{R}$ of the reals. We let $\mathrm{L}^{n}(E, F)$ denote the space of all continuous $n$-linear mappings from $E^{n}$ into $F$.

In [1] Keller has introduced a new method in the study of the differential calculus in locally convex spaces. He has used the topology of simple convergence in $L^{n}(E, F)$ in order to define the $n$th derivatives. The notions of continuity of these derivatives are based on the stronger convergence structures on $L^{n}(E, F)$. In this paper we shall apply this idea to the case of Fréchet spaces.

In the case of Banach spaces it is well known that the Banach fixed point theorem plays an important role in the proof of the inverse mapping theorem. This relies on the properties of the topology of uniform convergence on bounded subsets.

Therefore we shall find a convergence structure on $L^{n}(E, F)$ such that we can use the fixed point theorems of Sadovskii [3]. In this paper

Received 27 February 1981. The author wishes to thank the referee for valuable suggestions and for pointing out errors. 
we shall use the degree theory of limit compact vector fields. This theory was introduced by Sadovski i in [3].

In the case of Hausdorff measure of noncompactness ( $c f$. Example 1.1) our inverse mapping theorem is similar to Yamamuro's theorem in [4], but we still cannot compare them in detail. In the case of Kuratowski measure of noncompactness ( $c f$. Example 1.2) our theorem is clearly convenient for application to the problems concerned with the $\alpha$-ball-contractions.

This paper consists of four sections. In the first section we shall introduce the notations and definitions of measure of noncompactness and the topological degree of limit-compact vector fields and study the class of $M$-bounded operators. The second section is devoted to defining the convergence structures on $L^{n}(E, F)$ and studying their properties. In the third section we define the $c^{n}$-differentiable mappings, and we shall give the inverse mapping theorem in the last section.

Throughout the paper we shall adhere to the following list of notations.

$R$ : the field of real numbers

$\mathbb{C}$ : the field of complex numbers

$E, F, G:$ Fréchet spaces over $\mathbb{K}(\mathbb{K}=\mathrm{R}$ or $\mathbb{C})$

$V(E), V(F)$ : families of all open convex balanced neighborhoods of 0 in $E, F$

$A^{n} \equiv A \times \ldots \times A \quad(n$ times $)$

$\vec{A}$ or cl $A$ : closure of $A$

$\overline{c o} A$ : closure of the convex hull of $A$

$\partial A$ : boundary of $A$

$A \backslash B=\{x \in A: x \notin B\}$

$A+B=\{x+y: x \in A$ and $y \in B\}$

$t A=\{t x: x \in A\}$

$I$ : the identity mapping on $E$

$2^{E}$ : the family of all subsets of $E$.

\section{Measure of noncompactness and $M$-bounded mappings}

In this section we shall introduce the notations and definitions of measure of noncompactness and the topological degree of limit-compact 
vector fields, and study some basic properties of $M$-bounded mappings.

DEFINITION 1.1. Let $P$ be a nonvoid set and $P$ be the set of all mappings of $P$ into $[0, \infty]$. The order, addition and multiplication are defined on $P$ as usual $(0 \cdot \infty=0)$.

Let $h \in P$; we shall say that $h$ is finite if $h(p)<\infty$ for every $p$ in $P$.

DEFINITION 1.2. Let $P$ be as in Definition 1.1 , and $L$ be a mapping of $2^{E}$ into $P$; then $L$ is called a measure of noncompactness on $E$, if for every $A, B \in 2^{E}$ we have:

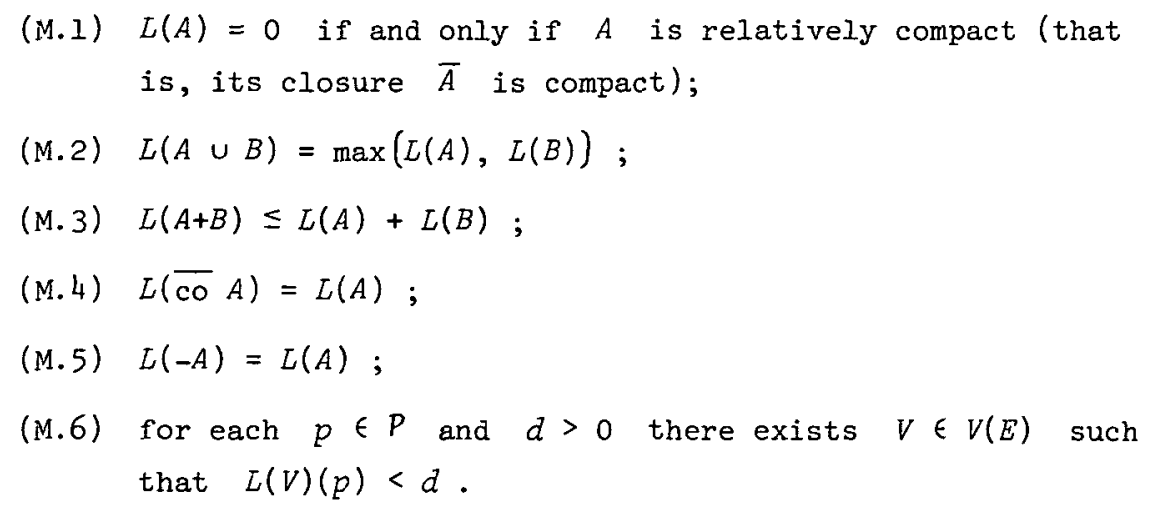

In [3] Sadovski $i$ has given many examples of measure of noncompactness (cf. $\$ 1.2$ of [3]). By Theorem 1.2.3 in [3] we have the two following useful examples of measures of noncompactness.

EXAMPLE 1.1. Let $P$ be a family of continuous seminorms on $E$ such that $P$ defines the topology of $E$. For each $A \in 2^{E}$ and each $p \in P$ we write

$$
\begin{aligned}
& L(A)(p)=\inf \left\{\text { there exist } A_{1}, \ldots, A_{k} \in 2^{E}\right. \text { such that } \\
& \left.r>0: \sup \left\{p(x-y): x, y \in A_{j}\right\}<r \text { for all } j \text { and } A \subset U_{I}^{k} A_{j}\right\}
\end{aligned}
$$

where inf $\Phi=\infty$.

Then $L$ is a measure of noncompactness on $E$ and is called the $P$-Hausdorff measure of noncompactness on $E$.

EXAMPLE 1.2. Let $E$ be a Fréchet space and $d$ be a translation 
invariant metric on $E$ such that $d$ is compatible with the topology of $E$ and $\{x: d(0, x)<r\}$ is convex and balanced for every $r>0$. For each $A$ in $2^{E}$ we write $L(A)=\inf \left\{\right.$ there exist $A_{1}, \ldots, A_{k} \in 2^{E}$ such that

$$
\left.r>0: \sup \left\{d(x, y): x, y \in A_{j}\right\}<r \text { for all } j \text { and } A \subset \underset{l}{k} A_{j}\right\} \text {. }
$$

Then $L$ is a measure of noncompactness on $E$ and is called the d-Kuratowski measure of noncompactness on $E$. If $E$ is a Banach space and $d(x, y)=\|x-y\|$, where \|\| is the norm on $E, L$ is called the norm-Kuratowski measure of noncompactness on $E$.

Throughout this paper we assume that there exist a fixed set $P$ and the given measures of noncompactness $L, M, N$ on $E, F, G$ respectively, as in Definition 1.2.

DEFINITION 1.3. Let $A \in 2^{E}$ and $f$ be a mapping of $A$ into $E$ and $B \subset A$; we write

$$
k(L, M, B, f)=\inf \{k>0: M(f(C)) \leq k L(C) \text { for every } C \subset B\} .
$$

If $M(f(A))$ is finite, $f$ is called a M-bounded mapping on $A$.

REl.ARK I.I. Let $D$ be a nonvoid open subset of $E$ and $f$ be a continuous $L$-bounded mapping of $\bar{D}$ into $E$ such that

$$
k(L, L, \bar{D}, f)<k<1 \text {. }
$$

Let $A$ be the limit range of $f$ on $\vec{D}$ (cf. Definition 1.1 .3 in [3]). By definition, $A=\overline{\operatorname{co}} f(A \cap \bar{D})$. Thus by (M.4), (M.2) and Definition 1.3, we have

$$
L(A)=L(f(A \cap \bar{D})) \leq k L(A \cap \bar{D}) \leq k L(A) .
$$

Because $L(f(\bar{D}))$ is finite and $L(f(A \cap \bar{D})) \leq L(f(\bar{D}))$, from (1.1) it follows that $L(f(A \cap \bar{D}))$ and $L(A)$ are finite and $L(A) \leq k L(A)$. But $k<1$; it follows that $L(A)=0$. Then, by (M.I), $A$ is compact. Therefore, by Definition 1.1.3 in [3], $f$ is a limit-compact mapping on $\bar{D}$.

Let $q \in E \backslash(I-f)(D)$ and put $f_{q}(x)=f(x)+q$ for every $x \in \bar{D}$. 
Because $f_{q}(A) \subset f(A)+\{q\}$ for every $A \in 2^{E}$, by properties of measure of noncompactness we see that $f_{q}$ is a continuous $L$-bounded mapping on $\bar{D}$ and $k\left(L, L, \bar{D}, f_{q}\right)<k<1$. Therefore $f_{q}$ also is a limit-compact mapping on $\bar{D}$ and for every $x \in \partial D, f_{q}(x) \neq x$.

Let $R=E ;$ by Definition 3.1 .4 of [3], the rotation of $\left(I-f_{q}\right)$ on $\bar{D}$ with respect to $R$ is defined and denoted by $\gamma\left(I-f_{q}, \bar{D}\right)$.

We shall write $\operatorname{deg}(I-f, D, q)=\gamma\left(I-f_{q}, D\right)$ and $\operatorname{deg}(I-f, D, q)$ is called the topological degree of $(I-f)$ at $q$ on $D$. By the results in $\$ 3.0$ and $\$ 3.2$ of [3], we have the following properties of the topological degree :

(D.I) if $q \in D$, then $\operatorname{deg}(I, D, q)=1$;

(D.2) if $\operatorname{deg}(I-q, D, q) \neq 0$ there exists $x \in D$ such that

$$
(I-f)(x)=q ;
$$

(D.3) if $D_{1}, \ldots, D_{k}$ are pairwise disjoint open subsets of $D$ such that $q \in E \backslash(I-f)\left(\bar{D} \backslash \begin{array}{cc}k \\ \cup \\ l\end{array} D_{j}\right)$, we have

$$
\operatorname{deg}(I-f, D, q)=\sum_{1}^{k} \operatorname{deg}\left(I-f, D_{j}, q\right)
$$

(D.4) let $E$ be a continuous mapping of $[0,1] \times \bar{D}$ into $E$ such that

$$
\begin{aligned}
& \text { (i) there exists a positive number } k<1 \text { such that } \\
& L(F([0,1] \times B)) \leq k L(B) \text { for every } B \subset \bar{D}, \\
& \text { (ii) } L(F([0,1] \times \bar{D})) \text { is finite; } \\
& \text { let } q \in E \backslash(x-F(t, x):(t, x) \in[0,1] \times \partial D\} ; \text { then } \\
& \operatorname{deg}(I-F(0, \cdot), D, q)=\operatorname{deg}(I-F(1, \cdot), D, q) .
\end{aligned}
$$

The properties (D.I), (D.2) and (D.3) follow directly from Theorems $3.0 .8,3.2 .6$ and 3.2 .5 in [3] respectively. Let $F$ and $q$ be as in (D.4); we put 


$$
E_{q}(t, x)=F(t, x)+q \text { for every }(t, x) \in[0,1] \times \bar{D} .
$$

Arguing as above we can show that $F_{q}$ is a limit-compact mapping on $[0,1] \times \bar{D}$. Then, by Theorem 3.2.2 in [3], we have (D.4).

Now we shall establish some basic properties of $M$-bounded mappings.

PROPOSITION 1.1. Let $A \in 2^{E}, B \in 2^{F}, f$ and $g$ be two mappings of $A$ into $B$, and $h$ be a mapping of $B$ into $G$. Suppose that $k(L, M, A, f), k(L, M, A, g)$ and $k(M, N, B, h)$ are finite. Then we have

(i) $k(L, M, A, f+g) \leq k(L, M, A, f)+k(L, M, A, g)$;

(ii) $k(L, N, A, h \circ f) \leq k(L, M, A, f) k(M, N, B, h)$;

(iii) if $f$ is M-bounded or $h$ is $N$-bounded then $h \circ f$ is $N$-bounded;

(iv) if $E=F=G, k(L, L, A, f)<1$, and $f$ is L-bounded: and suppose that $(I-f)$ is a one-to-one mapping of $A$ onto $B$ and $h=(I-f)^{-1} ;$ then

$$
k(L, L, B, h) \leq(1-k(L, L, A, f))^{-1} ;
$$

(v) Let $A, B, f$ and $h$ be as in (iv). Suppose that $A$ is closed and $f$ is continuous on $A$. Then $h$ is continuous on $B$.

Proof. Because $(f+g)(C) \subset f(C)+g(C)$ ana $N(h \circ f(C)) \leq k L(f(C))$ for every $C \in 2^{E}$ and every $k>k(M, N, B, h)$, we have (i) and (ii).

Since $N(h \circ f(A)) \leq \min \{N(h(B)), k(M, N, B, h) M(f(A))\}$, we have (iii).

Let $k \in] k(L, L, A, f), I[, D$ be a subset of $B$ and we put $D_{1}=h(D)$. Because $(I-f)\left(D_{1}\right)=D$ we have $D_{1} \subset D+f\left(D_{1}\right)$. Then $L\left(D_{1}\right) \leq L(D)+L\left(f\left(D_{1}\right)\right) \leq L(D)+k L\left(D_{1}\right)$. Hence it follows that

$$
L(h(D))=L\left(D_{1}\right) \leq \frac{1}{1-k} L(D)
$$

Therefore $k(L, L, B, h) \leq \frac{1}{1-k}$, which completes the proof of (iv). 
Now let $\left\{y_{j}\right\}$ be a sequence converging to $y$ in $B$. Put $D=\left\{y_{j}\right\} \cup\{y\}$. Then $D$ is compact and $D_{1}=h(D)=(I-f)^{-1}(D)$ is closed.

Because $L(D)=0$, by $(1.2), L\left(D_{1}\right)=0$. Hence it follows that $D_{1}$ is compact. Put $x_{j}=h\left(y_{j}\right)$ and let $x$ be an adherent point of $\left\{x_{j}\right\}$; that is, $x$ is the limit of a subsequence of $\left\{x_{j}\right\}$. By the continuity of $f,(I-f)(x)=y$. But $(I-f)$ is one-to-one: it follows that $\left\{x_{j}\right\}$ has an unique adherent point $x$, which is its limit. It is clear that $h(y)=x$. Therefore $h$ is continuous at $y$. Then $h$ is continuous on $B$. /

PROPOSITION 1.2. Let $f$ be a mapping of $[0,1] \times[0,1] \times A$ into $F$, where $A \in 2^{E}$. Suppose that:

(a) $f(t, \cdot)$ is M-bounded on $A$ for every $t \in[0,1] \times[0,1]$;

(b) there exists a real number $k$ such that $k(L, M, A, f(t, \cdot))$ is in $[0, k[$ for every $t \in[0,1] \times[0,1]$;

(c) for each $U \in V(F)$ there exists $d>0$, such that for every $s, t \in[0,1] \times[0,1]$ and $\|s-t\|<d$, and for every $x \in A$ we have

$$
(f(t, x)-f(s, x)) \in U
$$

Then we have

(i) $M(f([0,1] \times[0,1] \times A))$ is finite,

(ii) $M(f([0,1] \times[0,1] \times B)) \leq k L(B)$ for every $B \subset A$,

(iii) for each $x \in A$ let $g(x)$ be the Riemann integral

$$
\int_{0}^{1} \int_{0}^{1} f(t, s, x) d t d s \text {. }
$$

Then $g$ is M-bounded on $A$ and

$$
k(L, M, A, g) \leq k .
$$

Proof. Let $B$ be a subset of $A$, let $U \in V(F)$, then by condition (c) there exist $t_{1}, \ldots, t_{k}$ in $[0,1] \times[0,1]$ such that 


$$
f([0,1] \times[0,1] \times B) \subset U_{1}^{k} f\left(\left\{t_{j}\right\} \times B\right)+U .
$$

Thus, by (M.2), (M.3) and (M.6), it follows that for each $p \in P$ and $d>0$, there exist $t_{1}, \ldots, t_{k}$ in $[0,1] \times[0,1]$ such that

$$
M(f([0,1] \times[0,1] \times B))(p) \leq \max _{j}\left(M\left(f\left(\left\{t_{j}\right\} \times B\right)\right)(p)\right)+d .
$$

Hence it follows from (a) and (b) that (i) and (ii) hold.

Let $B \subset A$; we have $g(B) \subset \overline{c o} f([0,1] \times[0,1] \times B)$. Thus, by (i), (ii), (M.2), and (M.4), we have (iii). //

PROPOSITION 1.3. Let $V \in V(E)$ and $f$ be a continuous linear mapping of $E$ into $E$. Assume that

(a) $f$ is L-bounded on $\vec{V}$ and $k(L, L, \bar{V}, f)<1$,

(b) $f(\bar{V}) \subset \frac{1}{4} \bar{V}$.

Then we have

(i) $(I-f)(\bar{V}) \supset \frac{1}{4} \bar{V}$,

(ii) $(I-f)$ is a homeomorphism of $E$ onto $E$,

(iii) $k\left(L, L, \frac{1}{4} \bar{V},(I-f)^{-1}\right) \leq(1-k(L, L, \bar{V}, f))^{-1}$.

Proof. (i) For each $(t, x) \in[0,1] \times \bar{V}$ we put

$$
F(t, x)=t f(x) \text {. }
$$

Then for every $A \subset \bar{V}$ we see that $F([0,1] \times A)$ is contained in $\overline{c o}(f(A) \cup\{0\})$. Let $k \in] k(L, L, \bar{V}, f), I[$; then, by (a), $L F([0,1] \times \bar{V})$ is finite and

$$
L(F([0,1] \times A)) \leq k L(A) \text { for each } A \subset \bar{V} .
$$

On the other hand, for each $q \in \frac{1}{4} \bar{V}$ and $x \in \bar{V}$, by (b), we see that for each $t \in[0,1], q+F(t, x) \in \frac{1}{2} \bar{V}$. But $\frac{1}{2} \bar{V}$ is contained in $V$. Hence it follows that for every $(t, x)$ belonging in $[0,1] \times \partial V$ we have (1.3)

$$
x-F(t, x) \neq q \text {. }
$$

Then by (D.4) and (D.I) we have

$$
\begin{aligned}
\operatorname{deg}(I-f, V, q) & =\operatorname{deg}(I-F(1, \cdot), V, q)=\operatorname{deg}(I-F(0, \cdot), V, q) \\
& =\operatorname{deg}(I, V, q)=1 .
\end{aligned}
$$


Then by (D.2) there exists $x \in V$ such that $(I-f)(x)=q$. Therefore $(I-f)(\bar{V})=\frac{1}{4} \bar{V}$.

(ii) We shall show that $(I-f)$ is one-to-one. Indeed let $x \in E$ such that $(x-f(x))=(I-f)(x)=0$; hence $(I-f)(t x)=0$ for every real number $t$. Put $A=\mathrm{R} x$; by (1.3), $A \cap \partial V=\emptyset$. Then $A \subset V$ and $f(y)=y$ for every $y \in A$; hence $f(A)=A$. But, by $(a), L(f(A))$ is finite and

$$
L(A)=L(f(A)) \leq k L(A) .
$$

It follows that $L(A)$ is finite. Because $k<1,(1.4)$ implies that $L(A)=0$ and $A$ is relatively compact. Hence it follows that $x=0$, which implies that $(I-f)$ is one-to-one. Now, by (i) and by Proposition 1.1, $(I-f)$ is a homeomorphism of $E$ onto $E$.

(iii) By Proposition 1.1 we have (iii). //

Let $f$ be a toplinear isomorphism of $E$ onto $F$, that is $f \in L(E, F)$ and $f^{-1} \in L(F, E), f$ is said to be bibounded if there exist $V \in V(E)$ and $W \in V(F)$ such that $M(f(V)), L\left(f^{-1}(W)\right)$, $k(L, M, \bar{V}, f)$ and $k\left(M, L, \bar{W}, f^{-1}\right)$ are finite. Let $H(E, F)$ denote the set of all bibounded toplinear isomorphisms of $E$ onto $F$.

PROPOSITION 1.4. Let $g \in H(E, F), V \in V(E)$. Then there exist $U \in V(F)$ and $r>0$ such that for each continuous linear mapping $h$ of $E$ into $F$ which satisfies the following conditions,

(a) $h$ is M-bounded on $\bar{V}$ and $h(\bar{V}) \subset U$,

(b) $k(L, M, \bar{V}, h)<r$,

we have

(i) $(g-h)(\bar{V}) \supset \bar{U}$,

(ii) $(g-h) \in \not \rightarrow(E, E)$,

(iii) $k\left(M, L, \bar{U},(g-h)^{-1}\right) \leq 2 k\left(M, L, \bar{U}, g^{-1}\right)$.

Proof. Let $U \in V(F)$ be such that $k\left(M, L, \vec{U}, g^{-1}\right)$ is finite and $g^{-1}(\bar{U})$ is contained in $\frac{1}{4} V$, and let $\left.r \in\right] 0, \frac{1}{2}\left(1+k\left(M, L, \bar{U}, g^{-1}\right)\right)^{-1}[$. Now if $h$ satisfies conditions $(a)$ and $(b)$, we have 
(a') $g^{-1} \circ h$ is L-bounded on $\bar{V}$ and $k\left(L, L, \bar{V}, g^{-1} \circ h\right)<\frac{1}{2}$, (b') $\left(g^{-1} \circ h\right)(\bar{V}) \subset \frac{1}{4} V$.

By Proposition 1.3 it follows that $\left(I-\left(g^{-1} \circ h\right)\right)$ is a homeomorphism of $E$ onto $E$ and

$$
\left(I-\left(g^{-1} \circ h\right)\right)(\bar{V}) \supset \frac{1}{4} \bar{V} \supset g^{-1}(\bar{U})
$$

and

$$
\begin{aligned}
& k\left(L, L, \frac{1}{4} \bar{V},\left(I-\left(g^{-1} \circ h\right)\right)^{-1}\right) \leq\left(1-k\left(L, L, \bar{V}, g^{-1} \circ h\right)\right)^{-1} \leq 2 \\
& \text { Because }(g-h)=g \circ\left(I-\left(g^{-1} \circ h\right)\right), \text { we have }(i),(i i) \text { and (iii). // }
\end{aligned}
$$

\section{The topology on $B^{n}(E, F)$}

In this section we shall define the topology on $B^{n}(E, F)$ and study its basic properties.

DEFINITION 2.1. Let $B^{n}(E, F)$ denote the set of all continuous $n$-linear mappings $T^{\text {of }} E^{n}$ into $F$ satisfying the following conditions;

(B.I) there exists $V \in V(E)$ such that for each $V \in V(E)$ we can find $U \in V(E)$ such that $T\left(\vec{V}^{n-1} \times U\right) \subset W$;

(B.2) there exists $V \in V(E)$ and a positive real number $k$ such that for every $y \in \vec{V}^{n-1}, T(y, \cdot)$ is $M$-bounded on $\bar{V}$ and $\sup \left\{k(L, M, \bar{V}, T(y, \cdot)): y \in \vec{V}^{n-1}\right\}<k$.

For each $T \in B^{n}(E, F)$ and each $V \in V(E)$ we put

$$
k(n, L, M, \bar{V}, T)=\sup \left\{k(L, M, \bar{V}, T(y, \cdot)): y \in \vec{V}^{n-1}\right\} .
$$

DEFINITION 2.2. Let $T \in B^{n}(E, F)$; then $T$ is said to be totally symmetrical if $T\left(x_{f(1)}, \ldots, x_{f(n)}\right)=T\left(x_{1}, \ldots, x_{n}\right)$ for every $\left(x_{1}, \ldots, x_{n}\right) \in E^{n}$ and every permutation $f$ of $\{1, \ldots, n\}$.

We put 


$$
L^{n}(E, F)=\left\{T \in B^{n}(E, F): T \text { is totally symmetrical }\right\} .
$$

If $n=1$ we write $L(E, F)$ instead of $L^{1}(E, F)$.

REMARK 2.1. Let $E$ and $F$ be two Banach spaces, \|\| and \|\|$^{\prime}$ be the norms on $E$ and $F, L$ and $M$ be the norm-Kuratowski measures of noncompactness on $E$ and $F \quad$ (cf. Example 1.2) and $V=\{x \in E:\|x\|<1\}$.

Let $T$ be a continuous n-linear mapping of $E$ onto $F$, then there exists a positive real number $k$ such that

(2.1) $\left\|T\left(x_{1}, \ldots, x_{n}\right)\right\|^{\prime} \leq k\left\|x_{1}\right\| \ldots\left\|x_{n}\right\|$ for all $x_{1}, \ldots, x_{n} \in E$.

It is clear that $T$ satisfies (B.I) and (B.2); thus $T \in B^{n}(E, F)$. We have just proved that $B^{n}(E, F)$ is the set of all continuous n-linear mapping of $E$ into $F$ in the case of Banach spaces.

DEFINITION 2.3. For each $V \in V(E), U \in V(F), f \in B^{n}(E, E)$ and $k>0$, we let $B(n, V, U, k)$ denote the set of all $T$ belonging in $B^{n}(E, F)$ and satisfying the following conditions:

(i) $T, V$ and $k$ satisfy the conditions (B.1) and (B.2);

(ii) there exists $W \in V(F)$. such that $T\left(\nabla^{n}\right)+W \subset U$.

We put

$$
B(n, f, V, U, k)=f+B(n, V, U, k) \text {. }
$$

In the case $n=1$ we write $B(V, U, k)$ and $B(f, V, U, k)$ instead of $B(1, V, U, k)$ and $B(1, f, V, U, k)$.

REMARK 2.2. Let $V \in V(E), U, U^{\prime}, U^{\prime \prime} \in V(F), r, s>0$ and $g \in B(n, V, U, r)$ such that $g\left(\vec{V}^{n}\right)+U^{\prime \prime} \subset U$. Then it is clear that

$$
\begin{gathered}
B\left(n, V, U \cap U^{\prime}, \min (r, s)\right) \subset B(n, V, U, r) \cap B\left(n, V, U^{\prime}, s\right), \\
B\left(n, g, V, \frac{1}{2} U^{\prime \prime}, r-k(n, L, M, \bar{V}, g)\right) \subset B(n, V, U, r) .
\end{gathered}
$$

DEFINITION 2.4. By the foregoing remark there exists an unique topology on $\dot{B}^{n}(E, F)$ such that $\{B(n, f, V, U, k): U \in V(F), k>0\}$ is a basis of neighborhoods of $f$ in this topology. We denote this topology by $\tau_{V, n}$. If $n=1$, we write $\tau_{V}$ instead of $\tau_{V, 1}$. 
Let $\eta_{V, n}$ denote the topology induced by $\tau_{V, n}$ on $L^{n}(E, F)$.

REMARK 2.3. The notions and the topology defined in Definitions 2.1 and 2.3 are not symmetrical with respect to coordinates $x_{1}, \ldots, x_{n}$ of $E^{n}$. But our aim is the study of the differentiability. In this problem all mappings are in $L^{n}(E, F)$ and for each $T \in L^{n}(E, F)$ the notions in Definition 2.1 become symmetrical with respect to coordinates $x_{1}, \ldots, x_{n}$ of $E^{n}$. We also have the symmetry of $\eta_{V, n}$.

REMARK 2.4. We assume that $E, F, L, M, V$ are as in Remark 2.1. By (2.1) we can show that $\tau_{V, n}$ is the topology of uniform convergence on $\vec{V}^{n}$. Therefore $\tau_{V, n}$ is the topology $\tau$ of uniform convergence on bounded subsets of $B^{n}(E, F)$. Furthermore if $W$ and $U$ are in $V(E)$ and $W \subset U$, then $\tau_{U, n}$ is finer than $\tau_{W, n} \cdot$ Hence it follows that $\tau_{W, n}$ is $\tau$ for every bounded neighborhood $W$ of 0 in $E$.

REMARK 2.5. Let $V \in V(E)$; we see that

$$
\{B(n, V, U, k): U \in V(E), k>0\}
$$

is a base of a filter $F_{V}$ on $L^{n}(E, F)$.

We assume that $L$ and $M$ are as in Examples 1.1 and 1.2. Let $t \in \mathbf{R}, B \in 2^{F}$ and $m$ be an integer such that $|t| \leq m$; we have $M(t B) \leq m M(B)$. Then if $T \in B^{n}(E, F)$, we have that $t T \in B^{n}(E, F)$ and $k(n, L, M, \bar{V}, t T) \leq m k(n, L, M, \bar{V}, T)$. Hence it follows that $t F_{V}=F_{V}$ for every $t \neq 0$.

Now let $v$ be the filter of all neighborhoods of 0 in $R$. It follows from the preceding result that $v \cdot F_{V}=F_{V}$. Then, as in $\$ 0.7$ of [1], one can find a unique separated convergence structure on $L^{n}(E, F)$ which is invariant with respect to translations and determined by the set $\left\{F_{V}: V \in V(E)\right\}$ of filters which converge to 0 . It is clear that this convergence structure is finer than the convergence structure $\theta$ defined 
in $\S 0.7$ of [1].

In this paper we shall use the classical terminology of topology instead of notions of convergence structure.

For each integer $n$ and $V \in V(E)$ we shall denote $\left(B^{n}(E, F), \tau_{V, n}\right)$ and $\left(L^{n}(E, E), \eta_{V, n}\right]$ by $B^{n}(E, F, V)$ and $L^{n}(E, F, V)$.

DEFINITION 2.5. Let $n$ be an integer,

$$
J=\left\{i_{1}, \ldots, i_{m}\right\} \subset\{1, \ldots, n\}
$$

such that $i_{1}<i_{2}<\ldots<i_{n}$. We put

$$
H_{i}= \begin{cases}E & \text { if } i \in J, \\ \{0\} & \text { if } i \notin J,\end{cases}
$$

and let $p_{J, n}$ denote the projection of $E^{n}$ onto $H_{1} \times \ldots \times H_{n} \cdot$ In some cases we shall identity $H_{1} \times \ldots \times H_{n}$ with $E^{m}$ and write $p_{J}$ instead of $P_{J, n}$

In the following lemma let $\left\{I_{1}, \ldots, I_{p}\right\}$ be a partition of $\{1, \ldots, n\}$ and $m_{j}$ be the cardinal of $I_{j}, j=1, \ldots, r$. We put $p_{j}=p_{I_{j}, n}$ and consider the following lemma.

LEMMA 2.1. Let $T \in L^{r}(E, G)$ and $W \in V(E)$ satisfying (B.I) and (B.2). Let $S_{i} \in L^{m}(E, F)$ and $V \in V(E)$ satisfying (B.I) and (B.2), and assume that $s_{i}\left(v^{m} i\right) \subset i$ for every $i=1, \ldots, r$. Put

$$
\begin{aligned}
& k=\min _{j}\left(k\left(m_{j}, L, M, \bar{V}, s_{j}\right)\right), \\
& R=T \circ\left(s_{1} \circ p_{1}, \ldots, s_{r} \circ p_{r}\right) .
\end{aligned}
$$

Now for every $V_{j} \in V(E)$ such that $V_{j} \subset V$ for every $j$ in $\{1, \ldots, r\}$, we have 
(i) $R$ and $V$ satisfy (B.1) and (B.2),

(ii) $R\left(\bar{V}_{1}^{m} 1 \times \ldots \times \bar{V}_{r}^{m}\right) \subset T\left(\vec{W}^{r-1} \times S_{j}\left(\bar{V}_{j}^{m} j\right)\right)$ for all $j=1, \ldots, r$,

(iii) $k(m, L, N, \bar{V}, R) \leq k \cdot k(r, M, N, \bar{V}, T)$.

Proof. Because $T$ is totally symmetrical and $S_{j}\left(\frac{m}{V}_{j}^{j}\right) \subset W$ for every $j$, we have $(i i)$. We can suppose that $k\left(m_{r}, L, M, \bar{V}, S_{r}\right)=k$, then by Definition 2.1 and Proposition 1.1 we have $k(L, N, \bar{V}, R(y, \cdot)) \leq k \cdot k\left(L, M, \bar{V}, S_{r}\left(p_{r}(y, 0), \cdot\right)\right)$ for all $y \in \vec{V}^{n-1}$. This implies (iii); thus we also have (i). //

PROPOSITION 2.1. Let $n, m_{j}, p_{j}$ be as in Lemma 2.1, let $V \in V(E)$ and $W \in V(F)$. Let $T, S_{j}$ be continuous mappings of a topological space $X$ into $L^{r}(E, G, W), L^{m}{ }^{j}(E, F, V)$ respectively. Suppose that $(T(x), W)$ and $\left(S_{j}(x), V\right)$ satisfy the conditions (B.I) and (B.2) for every $x \in X$ and $j=1, \ldots, r$. For each $x \in X$ we put

$$
R(x)=T(x) \circ\left(S_{1}(x) \circ p_{1}, \ldots, S_{p}(x) \circ p_{p}\right) .
$$

Then for each $a \in X$ there exist $U \in V(E)$ and a neighborhood $Y$ of $a$ in $X$ such that the restriction $h \mid y$ of $h$ on $y$ is a continuous mapping of $Y$ into $B^{m}(E, G, U)$.

Proof. For the sake of simplicity we carry out the proof of this proposition in the special case $r=2$. The general case can be dealt with using essentially the same arguments.

Let $a \in X$; we choose $U \in V(E)$ such that $U \subset V$ and

$$
S_{1}(a)\left(\bar{U}^{m}\right) \cup S_{2}(a)\left(\bar{U}^{m}\right) \subset \frac{1}{4} W
$$

Let $Y$ be a neighborhood of $a$ in $X$ such that (2.2) $S_{i}(x) \in B\left(m_{1}, V, S_{i}(a), \frac{1}{4} W, 1\right)$ for every $x \in Y, i=1,2$. Then if $x, y \in Y$ and $i \in\{1,2\}$ we have 


$$
S_{i}(x)\left(\bar{U}^{m}\right) \subset \frac{1}{2} W
$$

$$
\left[S_{i}(x)-S_{i}(y)\right]\left(\bar{U}^{m} i\right) \subset W
$$

$$
k\left(m_{i}, L, M, \bar{U}, s_{i}(x)\right) \leq k_{i}=1+k\left(m_{i}, L, M, \bar{v}, S_{i}(a)\right) .
$$

On the other hand we have

$$
\text { (2.6) } \begin{aligned}
R(x)-R(y)=T(x) \circ\left(S_{1}(x)\right. & \left.\circ p_{1},\left[S_{2}(x)-S_{2}(y)\right] \circ p_{2}\right) \\
& +T(x) \circ\left(\left[S_{1}(x)-S_{1}(y)\right] \circ p_{1}, S_{2}(y) \circ p_{2}\right) \\
& +(T(x)-T(y)) \circ\left(S_{1}(y) \circ p_{1}, S_{2}(y) \circ p_{2}\right) .
\end{aligned}
$$

By (2.3), (2.4), (2.5), (2.6) and Lemma 2.1 we see that $R \mid Y$ is a continuous mapping of $Y$ into $B^{m}(E, G, U)$. //

We consider now the last proposition of this section.

PROPOSITION 2.2. Let $\varphi: H(E, F) \rightarrow H(E, F)$,

$$
\varphi(g)=g^{-1}
$$

Let $g \in H(E, E), V \in V(E)$ be given. Then there exist $r>0$ and $U \in V(F)$ such that

(i) $B(g, V, U, r) \subset H(E, F)$,

(ii) $\varphi \mid B(G, V, U, r)$ is continuous from $\left(B(g, V, U, r), \tau_{V}\right)$ into $\left(H(F, E), \tau_{U}\right)$.

Proof. Let $U$ and $r$ be as in Proposition 1.4; then for each $f$ in $B(g, V, U, r)$ we have

$$
\begin{gathered}
f \in H(E, F), \\
\varphi(f)(\bar{U}) \subset \bar{V}, \\
k(M, L, \bar{U}, \varphi(f)) \leq 2 k(M, L, \bar{U}, \varphi(g)) .
\end{gathered}
$$

On the other hand for each $f, h \in B(g, V, U, r)$ we have

$$
\varphi(f)-\varphi(h)=\varphi(h) \circ(h-f) \circ \varphi(f) .
$$

Thus by (2.8), (2.9) and Proposition 1.1, $\varphi \mid B(g, V, U, r)$ is continuous from $\left(B(g, V, U, r), \tau_{V}\right)$ into $\left(H(F, E), \tau_{U}\right) . / /$ 


\section{Functions of class $c^{n}$}

By a similar method to that used in $\$ 2$ of [1] we shall define notions of differentiability, but we use here the topology $\tau_{V, n}$ instead of the convergence structure $\theta$ defined in [1]. In this section $X$ is a given open subset of $E$, and $B^{0}(E, F)=L^{0}(E, F)=F$ and $L^{n}(E, F)$ is defined as in Definition 2.2.

DEFINITION 3.1. A function $f: X \rightarrow B^{n}(E, F)$ will be called weakly $m$-times differentiable if there exist functions

$$
D^{k} f: X \rightarrow B^{n+k}(E, E), \quad k=0,1, \ldots, m \text {, }
$$

such that $D^{0} f=f$ and for each $x \in X$, each $h \in E$ and each $k \in\{0,1, \ldots, p-1\}$ we have

$$
\lim _{\substack{t \rightarrow 0 \\ t \in \mathbb{K}}} t^{-I}\left(D^{k} f(x+t h)-D^{k} f(x)\right)=D^{k+1} f(x) h
$$

where the limit exists in $F$ if $n=k=0$, and with respect to the topology of simple convergence in $B^{n+k}(E, E)$ if $n+k>0$. If $k=1$, we shall write $f^{\prime}$ instead of $D f$ in some cases.

By Theorem 2.4.0 of [1] we can use only the totally symmetrical $n$-linear mappings in order to define the functions of class $c^{n}$ as follows.

DEFINITION 3.2. Let $m \geq 1$. A function $f: X \rightarrow L^{n}(E, F)$ is said to be differentiable of class $c^{m}$ or $c^{m}$-differentiable if $f$ is weakly $m$-times differentiable and for each $k \in\{0,1, \ldots, m\}$ and each $x \in X$ there exist $V \in V(E)$ and a neighborhood $Y$ of $x$ in $X$ such that $D^{k} f \mid y$ is continuous from $y$ into $F$, if $n+k=0$ and into $L^{n+k}(E, F, V)$ if $n+k>0$.

If $f$ is of class $c^{m}$ for every integer $m, f$ is said to be differentiable of class $c^{\infty}$, or $c^{\infty}$-differentiable.

REMARK 3.1. As in Remark 2.5 we see that if $f$ is weakly $m$-times 
differentiable (respectively, of class $c^{m}$ ) on $X$, then it is also weakly $m$-times differentiable (respectively of class $c_{\theta}^{m}$ ) in the sense of $\S 2$ of [1].

REMARK 3.2. Assume that $E$ and $F$ are Banach spaces, and $L$ and $M$ are norm-Kuratowski measures of noncompactness on $E$ and $F$. Then, by Remark 2.4, $f$ is of class $c^{n}$ on $x$ if and only if $f$ is n-times continuously Fréchet differentiable on $X$.

By Remark 3.1 and by Proposition 2.2.0 and Theorem 2.8.1 in [1] we have the following elementary properties of functions of class $c^{n}$.

PROPOSITION 3.1. Let $f$ be a $c^{n}$-differentiable mapping of $x$ into $F$. Let $x \in X$ and $Y$ be a neighborhood of $x$ in $X$ as in Definition 3.2, and $h \in E$ such that $\{x+t h: t \in[0,1]\} \subset y$. Then

(i) $\left|D^{n-1} f(x+h)-D^{n-1} f(x)\right| k=\int_{0}^{1} D^{n} f(x+t h)(h, k) d t$ for every $k \in E^{n-1}$;

(ii) we put

$$
R_{n} f(x, h)=f(x+h)-\sum_{0}^{n} \frac{1}{m !} D^{m} f(x)(h, \ldots, h) .
$$

Then there exists a continuous seminorm $p$ on $E$ such that $x+V \subset Y$, where $V=\{y \in E: p(y)<1\}$ and for each continuous seminorm $q$ on $F$, we can define a function $\theta_{p, q}: V \rightarrow \mathbf{R}$ such that

$$
\begin{gathered}
\lim _{k \rightarrow 0} \theta_{p, q}(k)=0, \\
q\left(R_{n} f(x, k)\right)=\theta_{p, q}(k)[p(k)]^{n} \text { for every } k \in V .
\end{gathered}
$$

It follows from (3.2) that if $k \in E$ and $R k r V$, then

$$
p(k)=0 \text { and } f(x+k)=f(x)+D f(x) h .
$$

Let us consider an example of $C^{n}$-differentiable mappings.

PROPOSITION 3.2. Assume that $E$ and $F$ are two Fréchet spaces over C . Let $g: X \rightarrow F$ be continuous and weakly n-times differentiable on 
$X$. Suppose that $g(X)$ is relatively compact. Then $g$ is of class $C^{n}$ on $X$.

Proof. Let $a \in X$ and $W \in V(E)$ such that $(a+3(n+1) \mathbb{W}) \subset X$. Let $m \leq n, x \in(a+2 W)$ and $u, h_{1}, \ldots, h_{n}, h \in 2 \bar{W}$, and $z, w \in \mathbb{C}$ such that $|z|,|w| \leq \frac{1}{2}$ and $\Gamma=\{z \in \mathbb{C}:|z|=I\}$ positively oriented. By the Cauchy formula for scalar analytic functions, the Hahn-Banach theorem and the induction we have

$$
\begin{gathered}
g(x+z h)=\frac{1}{2 \pi i} \int_{\Gamma} \frac{g(x+s h)}{s-z} d s, \\
g(x+z h)-g(x+w h)=\frac{z-\omega}{2 \pi i} \int_{\Gamma} \frac{g(x+s h)}{(s-z)(s-w)} d s,
\end{gathered}
$$

(3.4) $D^{m} g(x+z h)\left(h_{1}, \ldots, h_{m}\right)$

$$
=\frac{1}{(2 \pi i)^{m}} \int_{\Gamma} \cdots \int_{\Gamma} \frac{g\left(x+z h+s_{1} h_{1}+\ldots+s_{m} h_{m}\right)}{s_{1}^{2} \cdot s_{2}^{2} \ldots s_{m}^{2}} d s_{1} \ldots d s_{m} .
$$

It follows from (3.3) and (3.4) that

(3.5) $\left|D^{m} g(x+z h)-D^{m} g(x)\right|\left(h_{1}, \ldots, h_{m}\right)$

$$
=\frac{z}{(2 \pi i)^{m}} \int_{\Gamma} \cdots \int_{\Gamma} \frac{g\left(x+s_{1} h_{1}+\ldots+s_{m} h_{m}+s h\right)}{s_{1}^{2} \ldots s_{m}^{2}(z-s) s} d s_{1} \ldots d s_{m} d s .
$$

On the other hand we have

$$
\begin{aligned}
D_{g}^{m}(x)\left(h_{1}, \ldots,\right. & \left.h_{m-1}, 0\right) \\
& =\left[\frac{1}{(2 \pi i)^{m}} \int_{\Gamma} \ldots \int_{\Gamma} \frac{g\left(x+s_{1} h_{1}+\ldots+s_{m-1} h_{m-1}\right)}{s_{1}^{2} \ldots s_{m-1}^{2}} d s_{1} \cdots d s_{m-1}\right] \\
& \times \int_{\Gamma s_{m}^{2}} \frac{1}{2} d s_{m}=0 .
\end{aligned}
$$

Then 


$$
\begin{aligned}
& D^{m} g(x)\left(h_{1}, \ldots, h_{m-1}, w h_{m}\right) \\
& =D^{m} g(x)\left(h_{1}, \ldots, h_{m-1}, w h_{m}\right)+D^{m} g(x)\left(h_{1}, \ldots, h_{m-1}, 0\right) \\
& =\frac{1}{(2 \pi i)^{m}} \int_{\Gamma} \ldots \int_{\Gamma} \frac{g\left(x+s_{1} h_{1}+\ldots+s_{m} w h_{m}\right)-g\left(x+s_{1} h_{1}+\ldots+s_{m-1} h_{m-1}\right)}{s_{1}^{2} \ldots s_{m}^{2}} d s_{1} \ldots d s_{m} .
\end{aligned}
$$

Hence by (3.3) we have

$$
\begin{aligned}
D_{g(x)}^{m}\left(h_{1}, \ldots, h_{m-1}, w h_{m}\right) & \\
& =\frac{w}{(2 \pi i)^{m+1}} \int_{\Gamma} \ldots \int_{\Gamma} \frac{g\left(x+s_{1} h_{1}+\ldots+s_{m} s h_{m}\right)}{s_{1}^{2} \ldots s_{m-1}^{2} s_{m}^{2}(s-w) s} d s d s_{1} \ldots d s_{m} .
\end{aligned}
$$

We put $A=\overline{\operatorname{co}} g(X)$; then $A$ is bounded, which implies that for every $U \in V(F)$ then there exists $t>0$ such that $s A \subset U$ for every $s \in C$ and $|s| \leq t$. By (3.4) and (3.5), for every $x \in(a+2 W)$ and $y \in t U, t \in] 0, I[$ we have

$$
\begin{gathered}
D^{m} g(x)\left(\vec{W}^{m}\right) \subset A, \\
{\left[D^{m} g(x+y)-D^{m} g(x)\right]\left(\vec{W}^{m}\right) \subset t A .}
\end{gathered}
$$

Because $M(A)=0$, by $(3.6),(3.7)$ and $(3.8), D^{m} g(x)$ and $W$ satisfy the conditions (B.1) and (B.2) for every $x \in(a+W)$ and every $k>0$. Hence it follows from (3.8) that $D^{m} g \mid(a+W)$ is continuous from $(a+W)$ into $B^{m}(E, F, W)$.

Applying Theorem 2.4.0 of [1] we have $D^{m} g(x) \in L^{m}(E, F)$ for every $x \in(a+W)$, which completes our proof. //

By Proposition 1.2, in the preceding proposition we can replace the condition " $g(X)$ is relatively compact" by the following conditions:

(E.I) $M(z B)=|z| M(B)$ for each $z \in \mathbb{C}$ and $B \in 2^{F}$;

(E.2) $M(g(X))$ and $k(L, M, X, g)$ are finite;

(E.3) $g(X)$ is bounded in $F$.

Now we shall show some basic properties of $c^{n}$-differentiable mappings. 
PROPOSITION 3.3. Let $D$ be an open subset of $F$. If $f: X \rightarrow D$ and $g: D \rightarrow G$ are of class $c^{n}$, then $h=g \circ f$ is of class $c^{n}$ on $X$.

Proof. At first using the induction we shall show that $h$ is weakly $n$-times differentiable on $X$, and for every $x \in D$ and every integer $m \leq n, D^{m} h(x)$ can be written in the form

(3.9) $\sum_{i=0}^{m} \sum_{J \in J(n)} a_{J} D^{i} f(g(x)) \circ\left(D^{m} 1_{g(x)} \circ p_{J}, \ldots, D^{m^{i}} g(x) \circ p_{J_{i}}\right)$

where $J$ is a partition $\left\{J_{1}, \ldots, J_{i}\right\}$ of $\{1, \ldots, m\}$, and $m_{j}$ is the cardinal of $J_{j}$, and $a_{J} \in \mathbb{K}$.

In the case $m=1$, this assertion is clearly true. Now suppose that it is true for $m=r<n$. In order to prove this assertion in the case $m=x+1$ it is sufficient to show that for each partition $J=\left\{J_{1}, \ldots, J_{i}\right\}$ of $\{1, \ldots, m\}$ we have

$$
x \mapsto D^{i} f(g(x)) \circ\left(D^{m}{ }^{1} g(x) \circ p_{J_{1}}, \ldots, D^{m^{i}}(x) \circ p_{J_{i}}\right)
$$

is weakly differentiable and its derivative at $x$ can be written in the form

$$
\begin{aligned}
& D^{i+1} f(g(x)) \circ\left(D^{m^{1}} g(x) \circ p_{J_{1}}, \ldots, D^{m^{i}} g(x) \circ p_{J_{i}}, D g(x) \circ p_{\{i+1\}}\right) \\
& +D^{i} f(g(x)) \circ\left(D^{m_{1}^{+1}} g(x) \circ p_{J_{1}}\{i+1\}, D^{m_{2}} g(x) \circ p_{J_{2}}, \ldots, D^{m^{i}} g(x) \circ p_{J_{i}}\right) \\
& +\ldots+D^{i} f(g(x)) \circ\left(D^{m_{1}} g(x) \circ p_{J_{1}}, \ldots, D^{m_{i}^{+1}} g(x) \circ p_{J_{i} \cup\{i+1\}}\right) \text {. }
\end{aligned}
$$

But this follows from a simple differential calculus and Proposition 2.1. Then $D^{m} h(x)$ can be written in the form (3.9). Now applying Proposition 2.1 and by Theorem 2.4 .0 in [1] we see that $h$ is of class $c^{n}$ on $x$. /

Now let $f: X \rightarrow L^{j}(E, E), g: X \rightarrow L^{i}(E, E)$ be of class $C^{1}$ on $X$. Let $(I, J)$ be a partition of $\{1, \ldots, i+j-1\}$, such that the 
cardinal of $J$ is equal to $i$. For each $x \in X$ we put $h(x)=f(x) \circ\left(p_{I}, g(x) \circ p_{J}\right)$. It is clear that $h$ is weakly

differentiable on $X$ and

$$
D h(x)=D f(x) \circ\left(p_{I \cup\{i+j\}}, g(x) \circ p_{J}\right)+f(x) \circ\left(p_{I}, D g(x) \circ p_{J \cup\{i+j\}}\right) .
$$

By induction we have the following lemma.

LEMMA 3.1. Let $f, g: X \rightarrow L(E, E)$ be of class $c^{n}$. For each $x \in X$ we put $h(x)=f(x) \circ g(x)$. Then $h$ is weakly n-times differentiable on $X$ and for each $x \in X$ and $m \leq n$ we have

$$
D^{m} h(x)=\sum_{j} \sum_{(I, J)} a_{I, J} D^{j} f(x) \circ\left(p_{I}, D^{i} g(x) \circ p_{J}\right)+D^{m-1}[f(x) \circ D g(x)] .
$$

Furthermore by induction and by Proposition 2.1, we see that $h$ is of class $c^{n}$. (Here we remark that $p_{I}$ can be written in the form $\left(p_{\{1\}}, \ldots, p_{\{i\}}\right)$ if $\left.I=\{1, \ldots, i\}.\right\}$

Now we shall give a proposition about the differentiability of the mapping $\varphi$ (cf. Proposition 2.2).

PROPOSITION 3.4. Let $f: X \rightarrow L(E, E)$ be of class $C^{n}$ on $X$; suppose that $f(x) \in H(E, E)$ for every $x \in X$. Put

$$
g(x)=\varphi(f(x)) \text { for every } x \in X .
$$

Then $g$ is of class $c^{n}$ on $x$.

Proof. Let $x, y \in X$; we have

$$
g(y)-g(x)=g(y) \circ[f(x)-f(y)] \circ g(x) .
$$

Hence it follows that $g$ is weakly differentiable on $X$ and

$$
D g(x)=-g(x) \circ D f(x) \circ\left(p_{\{1\}}, g(x) \circ p_{\{2\}}\right) .
$$

Applying the arguments of Proposition 2.1, by Lemma 3.1 and induction we see that $g$ is of class $c^{n}$ on $x$.

\section{The inverse mapping theorem}

In this section we shall establish an inverse mapping theorem for 
$C^{n}$-differentiable mappings. At first let us consider the case of homeomorphisms.

THEOREM 1. Let $D$ be an open subset of $E$ and $f: D \rightarrow F$ be of class $C^{n}$ on $D$. Suppose that $f$ is a homeomorphism of $D$ onto an open subset of $F$, and there exists a $D$ such that $D f(a)$ is in $H(E, F)$. Then there exists an open neighborhood $X$ of $a$ in $D$ such that if $g=(f \mid X)^{-1}$ and $Y=f(X)$ then $g$ is of class $c^{n}$ on $y$ and for every $y \in Y$ we have $D g(y)=[D f(g(y))]^{-1}$.

Proof. By Proposition 1.4 we can find an open neighborhood $X$ of $a$ in $D$ such that $D f(x) \in H(E, F)$ for every $x \in X$. Fix an $y \in Y$; we shall show that $g$ is weakly differentiable at $y$ and

$$
D g(y)=[D f(g(y))]^{-1} \text {. }
$$

By Proposition 2.1 we can suppose that $E=F, y=g(y)=0$, and $f^{\prime}(0)=I$.

Let $p, V, \theta_{p, p}$ for $f$ and $x=0$ as in Proposition 3.1. Let $U \in V(E)$ such that $U \subset \frac{1}{3} V$ and $U \subset f(V)$ and

$$
\theta_{p, p}(h) \leq \frac{1}{3} \text { for every } h \in f^{-1}(U) \text {. }
$$

Now let $k \in U, t \in \mathbb{K}$ such that $0<|t|<1$. Then there exists $h_{t, k} \in V$ such that $g(t k)=h_{t, k}$. We shall show that

$$
p\left(t^{-1} h_{t, k}\right) \leq 1 \text { if } k \in U \text { and } 0<|t|<1 \text {. }
$$

Indeed if there exist $k \in U, t \in \mathbb{K}$ such that $0<|t|<1$ and $p\left(t^{-1} h_{t, k}\right)>1$, there exists $\left.s \in\right] 0,1\left[\right.$ such that $s p\left(t^{-1} h_{t, k}\right)=1$. But

$$
t k=f\left(h_{t, k}\right)-f(0)=f^{\prime}(0) h_{t, k}+R f\left(0, h_{t, k}\right)
$$

where

$$
R f(x, h)=f(x+h)-f(x)-D f(x) h .
$$

Because $D f(0)=I$, we have 


$$
t^{-1} h_{t, k}=k-t^{-1} R f\left(0, h_{t, k}\right)
$$

On the other hand, by (3.2) we have

$$
\begin{aligned}
p\left(s t^{-1} R f\left(0, h_{t, k}\right)\right) & =s t^{-1} p\left(R f\left(0, h_{t, k}\right)\right) \quad s t^{-1} p\left(h_{t, k}\right) \theta_{p, p}\left(h_{t, k}\right) \\
& \leq s p\left(t^{-1} h_{t, k}\right) \theta_{p, p}\left(h_{t, k}\right) \leq \theta_{p, p}\left(h_{t, k}\right) \leq \frac{1}{3} .
\end{aligned}
$$

Since $p(s k) \leq \frac{1}{3}$, by $(4.3)$ it follows that

$$
I=p\left(s t^{-1} h_{t, k}\right) \leq p(s k)+p\left(s t^{-1} R f\left(0, h_{t, k}\right)\right) \leq \frac{2}{3} \text {. }
$$

This contradiction shows (4.1). Now let $q$ be an arbitrary continuous seminorm on $E$, let $\theta_{p, q}$ be as in Proposition 3.1. We have as above

$$
q\left(t^{-1} R f\left(0, h_{t, k}\right)\right) \leq \theta_{p, q}\left(h_{t, k}\right)
$$

Since $\lim _{t \rightarrow 0} h_{t, k}=0$, by (3.1) we have $\lim _{t \rightarrow 0} t^{-1} R f\left(0, h_{t, k}\right)=0$. On the other hand, by (4.3) we have

$$
t^{-1}[g(t k)-g(0)]=[D f(0)]^{-1} k-t^{-1} R f\left(0, h_{t, k}\right) .
$$

Therefore $g$ is weakly differentiable at $y$ and $D g(y)$ is equal to $|D f(g(y))|^{-1}$. By Proposition 2.2, $g$ is of class $C^{\perp}$ on $Y$. Now if $n>1$, we put $h(y)=\varphi(D f(g(y)))$ for every $y \in Y$. Then by Propositions 3.3 and $3.4, h$ is of class $C^{l}$ on $Y$, hence $g$ is of class $C^{2}$ on $Y$. By induction $g$ is of class $C^{n}$ on $y$. //

We shall establish an inverse mapping theorem for vector fields, but first we need the following lemma.

LEMMA 4.1. Let $W$ be an open in $E, f=(I-g): W \rightarrow E$ be of class $C^{l}$ on $W$. Suppose that $k(L, L, W, g)<k<1$ and for every $a \in W, f^{\prime}(a) \in H(E, E)$ and $k(L, L, W, I-\varphi(D f(a)))<\frac{1-k}{1+k}$. Let $a \in W$; then there exist $U, V \in V(E)$ such that $a+V \subset W$ and for each $(t, q) \in[0,1] \times \bar{U}$ we have 
(i) $t[f(a+x)-f(a)]-(1-t) f^{\prime}(a)(x) \neq q$ for alz $x \in$ วV, or $t[f(y)-f(a)]-(1-t) f^{\prime}(a)(y-a) \neq q$ for alz $y \in a+\partial V$;

(ii) $f(x+a) \neq f(a)$ for $a Z z \quad x \in V \backslash\{0\}$.

Proof. Fix an $a \in W$; we have $S \in B(E, E)$ such that $k(L, L, W, S)<\frac{1-k}{1+k}$ and $\varphi\left(f^{\prime}(a)\right)=I+S$. Put $f_{1}=\varphi(D f(a)) \circ f$. Then $D f_{1}(a)=I$ and $f_{1}=I-g_{1}$, where $g_{1}=g+S \circ g-S$. By Proposition 1.1 we have

$$
\begin{aligned}
k\left(L, L, W, g_{1}\right) \leq k(L, L, W, g)+k(L, L, W, S \circ g) & +k(L, L, W, S) \\
& <k+\frac{(1-k) k}{1+k}+\frac{1-k}{1+k}=1 .
\end{aligned}
$$

We see that if the lemma holds for $f_{1}$, then it holds for $f$. Then we can suppose that $D f(a)=I$ and $a=f(a)=0$. By Proposition 3.1 we can choose $W^{\prime} \in V(E)$ such that $W^{\prime} \leftarrow W$ and $D f W^{\prime}$ is a continuous mapping from $W^{\prime}$ into $B\left(E, E, W^{\prime}\right)$ and (4.5) $f(x)=f^{\prime}(0) x=x$ for every $x \in W^{\prime}$ such that $\mathbb{K} x \subset W^{\prime}$.

Let $V \in V(E)$ such that $\bar{V} \subset W^{\prime}$ and

$$
\left(f^{\prime}(0)-f^{\prime}(x)\right) \in B\left(W^{\prime}, \frac{1}{4} W^{\prime}, \frac{1}{4}\right) \text { for all } x \in \bar{V} \text {. }
$$

By Proposition 3.1 we have

$$
g(x)=\int_{0}^{1}\left(f^{\prime}(0)-f^{\prime}(s x)\right) x d s \text { for all } x \in W^{\prime} .
$$

Put

$$
F=\{t f(x)-(1-t) x=x-t g(x):(t, x) \in[0,1] \times \partial V\}
$$

We shall show that $F$ is closed and does not contain 0 , which implies (i).

Now let $\left\{\left(t_{j}, x_{j}\right)\right\}$ be a sequence in $[0,1] \times \partial V$ such that $\left\{y_{j}\right\}$ converges to $y$ in $E$, where $y_{j}=x_{j}-t_{j} g\left(x_{j}\right)$. We can suppose that $\left\{t_{j}\right\}$ converges to $t$ in $[0,1]$. Put $A=\left\{x_{j}\right\}, B=\left\{y_{j}\right\} \cup\{y\}$ and $C=\overline{c o}(g(A) \cup\{0\})$. Since $x_{j}=y_{j}+t_{j} g\left(x_{j}\right)$, we see that $A \subset B+C$. Arguing as in the proof of Proposition 1.1 we see that $A$ is relatively 
compact. Let $x$ be an adherent point of $\left\{x_{j}\right\}$, then $x \in \partial V$. By Proposition 3.1, $f$ is continuous on $D$; thus $t f(x)+(1-t) x=y$. Then $E$ is closed.

Now suppose that there exists $(t, x) \epsilon[0,1] \times \partial V$ such that

$$
\begin{aligned}
t f(x)+(1-t) f^{\prime}(0) x & =(I-t g)(x)=x-t \int_{0}^{1}\left(f^{\prime}(0)-f^{\prime}(s x)\right) x d s \\
& =0 .
\end{aligned}
$$

If $\mathbb{K} x \subset W^{\prime}$, by (4.5), $f^{\prime}(0)=0$; hence $x=0$, which contradicts the condition $x \in \partial V$.

Then there exists $c \in \mathbb{K}$ such that $c x \in \partial W^{\prime}$; hence we have

$$
c x=\int_{0}^{I}\left(f^{\prime}(0)-f^{\prime}(s x)\right) t c x d s .
$$

By (4.6) it follows that $c x \in \frac{1}{4} \bar{W}^{\prime} \subset W^{\prime}$, which contradicts the condition $c x \in \partial W^{\prime}$. Then $F$ does not contain 0 .

(ii) Let $x \in V$ such that $f(x)=0$; we shall show that $x=0$. If $\mathbb{K} x \subset W^{\prime}$, then by (4.5), $f^{\prime}(0) x=x=0$. Now if there exists $t \in \mathbb{K}$ such that $t x \in \partial W^{\prime}$, we have

$$
t f(x)=t(I-g)(x)=t x-\int_{0}^{1}\left(f^{\prime}(0)-f^{\prime}(s x)\right) t x d s=0 .
$$

Arguing as in ( $i$ ) we can find a contradiction, which completes our proof. //

Applying Theorem 1 and Lerma 4.1 we can show the following inverse mapping theorem for vector fields.

THEOREM 2. Let $D$ be an open in $E, f=(I-g): D \rightarrow E$ be of class $C^{n}$ on $D$. Suppose that $0 \in D$ and $f(0)=0, f^{\prime}(0)=I, g$ is $L$-bounded on $D$ and $k(L, L, D, g)<1$. Then there exists an open neighborhood $X$ of 0 such that $X \subset D$ and $f \mid X$ is a homeomorphism of $X$ onto an open subset $Y$ of $E$. Put $h=(f \mid x)^{-1}$; then $h$ is of class $c^{n}$ on $y$ and for every $y \in Y$ we have

$$
h^{\prime}(y)=[D f(h(y))]^{-1} \text {. }
$$


Proof. By Proposition 2.2 we can choose $W, W^{\prime}, V$ and $U$ for $a=0$ as in Lemma 4.1 such that $\bar{U} \subset V$. Now let $p \in U$. By Lemma 4.1 and (D.4) we have

$$
\operatorname{deg}(f, V, p)=\operatorname{deg}\left(f^{\prime}(0), V, p\right)=\operatorname{deg}(I, V, p)=1 .
$$

By (4.6) and Remark 1.1, for each $a \in V$ and each $V^{\prime} \in V(E)$ such that $a+\bar{V}^{\prime} \subset W^{\prime}, g^{\prime}(a)$ is a limit-compact mapping on $a+\bar{V}^{\prime}$. By (4.6) and Proposition 3.1, $\left(I-\operatorname{tg}^{\prime}(a)\right) \in H(E, E)$, which implies that $\left(I-\operatorname{tg}^{\prime}(a)\right)(y-a) \neq 0$ for every $y \in a+\partial V^{\prime}$. Arguing as in Proposition 3.1, by (D.1) and (D.4) we have

$$
\begin{aligned}
\operatorname{deg}\left(f^{\prime}(a)(\cdot-a), a+V^{\prime},\right. & 0)=\operatorname{deg}\left(\left(I-g^{\prime}(a)\right)(\cdot-a), a+V^{\prime}, 0\right) \\
= & \operatorname{deg}\left(I(\cdot-a), a+V^{\prime}, 0\right)=\operatorname{deg}\left(I, a+V^{\prime}, a\right)=1 .
\end{aligned}
$$

Put $A=(f \mid \bar{V})^{-1}(\{p\})=((I-g) \mid \bar{V})^{-1}(\{p\})$. Arguing as in the proof of Lemma 4.1, we see that $A$ is compact. Then by Proposition 1.3 and by (ii) of Lemma 4.I, $A$ consists only of isolated points. Then $A=\left\{a_{1}, \ldots, a_{m}\right\}$.

By Lemma 4.1 we can choose $V^{\prime} \in V(E)$ such that $V^{\prime} \subset W^{\prime}$ and $\left\{a_{j}+V^{\prime}: j=1, \ldots, m\right\}$ is a family of pairwise disjoint open subsets of $V$ and for every $j \in\{1, \ldots, m\}$ we have, by (4.8),

$$
\begin{aligned}
\operatorname{deg}\left(f, a_{j}+V^{\prime}, p\right) & =\operatorname{deg}\left(f, a_{j}+V^{\prime}, f\left(a_{j}\right)\right)=\operatorname{deg}\left(I-\left(g+f\left(a_{j}\right)\right), a_{j}+V^{\prime}, 0\right) \\
& =\operatorname{deg}\left(f-f\left(a_{j}\right), a_{j}+V^{\prime}, 0\right) \\
& =\operatorname{deg}\left(f^{\prime}\left(a_{j}\right)\left(\cdot-a_{j}\right), a_{j}+V^{\prime}, 0\right)=1 .
\end{aligned}
$$

From (4.3) and (D.3) it follows that

$$
I=\operatorname{deg}(f, V, p)=\sum_{j} \operatorname{deg}\left(f, a_{j}+V^{\prime}, p\right)=m
$$

Hence it follows that for each $p \in U$ there exists an unique $x \in V$ such that $f(x)=p$. Put $Y=U$ and $X=V \cap f^{-1}(Y)$.

Then $f \mid X$ maps $X$ onto $Y$. Analogously $f \mid X$ also is an open mapping of $X$ onto $Y$. Therefore $f \mid X$ is a homeomorphism of $X$ onto $Y$. Now applying Theorem 1 , we have the desired results. //

REMARK 4.1. In Theorem 2 we can replace the condition $f^{\prime}(0)=I$ by the condition $f^{\prime}(0) \in H(E, E)$. This result relies on the study of the 
spectrum of $L$-bounded linear operators and will appear elsewhere.

DEFINITION 4.1 . Let be an open in $E, f: D \rightarrow F$ be of class $C^{l}$ on $D$. For each $x \in D$ put $R f(x, h)$ as in (4.2). We shall say that $f$ satisfies the condition (c) on $D$ if

(C) for each $x \in D$ and each $d>0$, there exists a neighborhood $X$ of $x$ in $D$ such that $k(L, M, X-x, R f(x, \cdot))<d$.

REMARK 4.2. If $E$ and $F$ are Banach spaces, $D$ is an open subset of $E, L$ and $M$ are the norm-Kuratowski measures of non-compactness on $E$ and $F$ and $f: D \rightarrow F$ is of class $C^{l}$. Then $f$ satisfies the condition $(C)$ on $D$.

In fact, let $x \in D, d>0$ and $V \in V(E)$ such that $x+V \subset D$. Let $h, k \in V$; we have

$$
\begin{aligned}
R f(x, h)-R f(x, k) & =f(x+h)-f(x+k)-D f(x)(h-k) \\
& =\int_{0}^{1}[D f(x+t(h-k))-D f(x)](h-k) d t .
\end{aligned}
$$

Thus

$$
\|R f(x, h)-R f(x, k)\| \leq \sup _{0 \leq t \leq 1}\|D f(x+t(h-k))-D f(x)\|\|h-k\|
$$

Therefore if $V$ is small enough, $k(L, M, V, R f(x, \cdot))<d$.

REMARK 4.3. Let $E, F, X, g, x, W$ be as in Proposition 3.2. By (3.5), $D g(x)(W)$ is relatively compact, then $R g(x, W)$ also is relatively compact; hence $k(L, M, W, R g(x, \cdot))=0$. Then $g$ satisfies the condition (C) on $X$. Since $D(T+g)=T+D g$ for every $T \in B(E, F)$, $R(T+g)(x, \cdot)=R g(x, \cdot)$. Then $T+g$ satisfies the condition (C) on $X$ for every $T \in B(E, F)$.

Now we can announce the inverse mapping theorem for the general case as follows.

THEOREM 3 (Inverse mapping theorem). Let $D$ be an open in $E$, $f: D+F$ be of class $C^{n}$ on $D$ and $a \in D$ such that $f^{\prime}(a) \in H(E, E)$. suppose that $f$ satisfies the condition (C) on $D$. Then there exists an open neighborhood $X$ of $a$ in $D$ such that $f \mid x$ is a homeomorphism of 
$X$ onto an open $Y$ of $F$. Put $g=(f \mid X)^{-1}$; then $g$ is of class $c^{n}$ and satisfies the condition (c) on $Y$. For every $y \in Y$ we have

$$
g^{\prime}(y)=\left[f^{\prime}(g(y))\right]^{-1}
$$

Proof. We can suppose that $a=f(a)=0$. Put $T=(D f(0))^{-1}$ and $h=T \circ f$; we have $h^{\prime}(0)=I$ and $h(0)=0$. For each $x \in D$ we have $h(x)=T(f(x)-f(0))=T(D f(0) x+R f(0, x))=(I-(T \circ R f(0, \cdot)))(x)$.

Since $f$ satisfies the condition $(C)$ and $T \in B(F, E)$, there exists an open neighborhood $D^{\prime}$ of 0 in $D$ such that

$$
k\left(L, L, D^{\prime}, T \circ R f(0, \cdot)\right)<1 \text {. }
$$

Then by Theorem 2, there exist an open neighborhood $X$ of 0 in $D^{\prime}$ such that $h \mid X$ is a homeomorphism of $X$ onto an open $U$ in $E$. Then $f \mid X$ is a homeomorphism of $X$ onto $y=T^{-1}(U)$. By Proposition 2.2, we can suppose that $f^{\prime}(x) \in H(E, F)$ for every $x \in X$. Thus by Theorem 1 , $g=(f \mid x)^{-1}$ is of class $C^{n}$ on $y$ and for every $y \in Y$ we have $g^{\prime}(y)=\left(f^{\prime}(g(y))\right)^{-1}$. Now we shall show that $g$ satisfies the condition (c) on $Y$.

Fix an $y \in Y$; for the sake of simplicity we suppose $y=0$ and consider $R g(0, \cdot)$. By $(4.4), R h^{-1}(0, \cdot)=-R f(0, \cdot) \circ h^{-1}$. Then

$$
R g(0, \cdot)=-R f(0, \cdot) \circ h^{-1} \circ T \text {. }
$$

Since $k\left(L, L, D^{\prime}, T \circ R f(0, \cdot)\right)<1$ and $h=I-T \circ R f(0, \cdot)$, by Froposition 1.1, $k\left(L, L, U, h^{-1}\right)$ is finite. Then by Proposition 1.1 and (4.9), $g$ satisfies the condition (c) at $y$. This completes the proof of the theorem. $\quad / /$

Let $S$ be a compact linear mapping of $E$ into $E$, that is, there exists $V \in V(E)$ such that $S(V)$ is relatively compact. It is well known that if $(I-S)(E)=E$, then it is a homeomorphism from $E$ onto $E$, and $(I-S)^{-1}=I+(I-S)^{-1} \circ S$. Because $(I-S)^{-1} \circ S$ is compact, we see that $(I-S) \in H(E, E)$. Then by Remark 4.3, Proposition 3.2 and Theorem 3 we have the following theorem. 
THEOREM 4 (The inverse mapping theorem for analytic vector fields). Let $E$ and $F$ be two Fréchet spaces over $\mathbb{C}$. Let $g$ be a continuous and weakly $n$-times differentiable from an open set $D$ in $E$ into $E$. Suppose that $g(D)$ is relatively compact and $\left(I-g^{\prime}(a)\right)$ is surjective for an $a \in D$. Then there exists an open neighborhood $X$ of $a$ in $D$ such that $f=(I-g) \mid X$ is a homeomorphism of $X$ onto an open $Y$ in $E$, and $f$ and $f^{-1}$ are of class $c^{n}$ on $X$ and $y$ repsectively and for each $y \in Y$ we have

$$
D f^{-1}(y)=\left[D f\left(f^{-1}(y)\right)\right]^{-1} \text {. }
$$

With the product topology $E \times F$ also is a Fréchet space. We shall identify $E \times\{0\}$ and $\{0\} \times F$ to be $E$ and $F$ respectively. Let $\mathrm{pr}_{1}$ and $\mathrm{pr}_{2}$ be the projections of $E \times F$ onto $E$ and $F$. Assume that there exists a measure of noncompactness $Q$ on $E \times F$ such that $\mathrm{pr}_{1}$ and $\mathrm{pr}_{2}$ are the elements of $B(E \times F, E \times F)$. Let $L$ and $M$ be the restrictions of $Q$ on $2^{E}$ and $2^{F}$. Because the projections are open, $L$ and $M$ are measures of noncompactness on $E$ and $F$.

Using the notions and arguments in $\$ 7$ of Chapter $V$ and $\$ 2$ of Chapter VI in [2], and applying Theorem 3 we have the following theorem.

THEOREM 5 (The implicit function theorem). Let $V \times U$ be an open set in $E \times F$ and $f: V \times U \rightarrow G$ be of class $C^{n}$, and $(a, b) \in V \times U$. Assume that $f$ satisfies the condition (C) on $V \times U$ and the partial derivative with respect to the second variable $D_{2} f(a, b) \in H(F, G)$ and $f(a, b)=0$. Then there exists a continuous mapping $g$ from $V_{0}$, an open neighborhood of $a$, into $U$ such that $g(a)=b$ and $f(x, g(x))=0$ for every $x \in V_{0}$.

If $V_{0}$ is taken to be sufficiently small, then $g$ is uniquely determined and is a $c^{n}$-differentiable mapping on $V_{0}$. 


\section{References}

[1] H.H. Keller, Differential calculus in locally convex spaces (Lecture Notes in Mathematics, 417. Springer-Verlag, Berlin, Heidelberg, New York, 1974).

[2] Serge Lang, Real cnalysis (Addison-Wesley, Reading, Massachusetts; Menlo Park, California; London; 1969).

[3] B.N. Sadovskii, "Limit-compact and condensing operators", Russian Math. Surveys 27 (1972), no. 1, 85-155.

[4] Sadayuki Yamamuro, "A differentiation in locally convex spaces", Bulz. Austral. Math. Soc. 12 (1975), 183-209.

Faculty of Mathematics,

University of Hochiminh City,

Hochiminh City,

vietNam. 